\title{
Thymidine synthase, thymidine phosphorylase, and excision repair cross-complementation group | expression as predictive markers of capecitabine plus cisplatin chemotherapy as first-line treatment for patients with advanced oesophageal squamous cell carcinoma
}

\author{
S Lee ${ }^{1,5}$, YH Park ${ }^{1,5}$, KH Kim', EY Cho', YC Ahn³, K Kim ${ }^{4}$, Y-M Shim ${ }^{4}$, JS Ahn', K Park' and Y-H Im, I \\ 'Division of Hematology-Oncology, Department of Medicine, Samsung Medical Center, Sungkyunkwan University School of Medicine, 50 Irwon-dong \\ Gangnam-gu, Seoul 135 - 7I 0, Korea; ${ }^{2}$ Department of Pathology, Samsung Medical Center, Sungkyunkwan University School of Medicine, Seoul, Korea; \\ ${ }^{3}$ Department of Radiation Oncology, Samsung Medical Center, Sungkyunkwan University School of Medicine, Seoul, Korea; ${ }^{4}$ Department of Thoracic \\ Surgery, Samsung Medical Center, Sungkyunkwan University School of Medicine, Seoul, Korea
}

BACKGROUND: Our purpose was to evaluate thymidine synthase (TS), thymidine phosphorylase (TP), and excision repair cross-complementation group I (ERCCI) expression as biomarkers for capecitabine and cisplatin (XP) combination chemotherapy in patients with metastatic oesophageal squamous cell cancer.

METHOD: A total of 113 patients with metastatic oesophageal squamous cell cancer were treated with XP chemotherapy at the Samsung Medical Center between 2003 and 2007, of whom 72 had available clinical data and paraffin blocks for immunohistochemistry of TS, TP, and ERCCI.

RESULTS: The median age of the 72 patients was 62 years. The overall response rate (RR) was $51.4 \%$. The median progression-free survival (PFS) and overall survival (OS) were 4.2 and 12.0 months, respectively. High expression of TS and TP was associated with a higher RR than was low expression of TS and TP (54.I vs 40.5\%, P=0.022). Strong ERCCI expression and a low TS score were identified as unfavourable independent risk factors for PFS (HR I0.7I, 95\% confidence interval (Cl) 2.I $-54.7, P=0.004$ for strong ERCCI expression; and HR 2.9, 95\% Cl I.0-7.9, P=0.044 for low TS score). Strong ERCCI expression was identified as an unfavourable independent risk factor for $\mathrm{OS}(\mathrm{HR} 3.73,95 \% \mathrm{Cl} 1.39-10.0, P=0.009)$.

CONCLUSION: These data indicate that expression of TS, TP, and ERCCI may be predictive markers for response and survival in patients with metastatic oesophageal squamous cell cancer receiving XP chemotherapy.

British Journal of Cancer (2010) I 03, 845-85I. doi:I0.1038/sj.bjc.660583I www.bjcancer.com

Published online 10 August 2010

(C) 2010 Cancer Research UK

Keywords: oesophageal cancer; capecitabine; cisplatin; thymidine synthase (TS); thymidine phosphorylase (TP); excesion repair cross-complementation group I (ERCCI)

Oesophageal cancer is the eight most frequent malignancy and the fourth highest cause of cancer-related mortality, with almost 500000 new patients diagnosed annually worldwide (Kamangar et al, 2006; Tebbutt et al, 2010). The most frequent histological type of oesophageal cancer is squamous cell carcinoma (SCCA), although the proportion of adenocarcinomas in western Europe and the United States is increasing to almost 50\%, with no difference in long-term outcomes between the two histological types. Oesophageal cancer is a highly virulent disease with a 5 -year survival rate of 10-15\% (Lenz et al, 1996; Bronckaers et al, 2009). At presentation, approximately $50 \%$ of patients show distant metastases and the remaining patients who initially present with locoregional disease will eventually develop distant metastases. There is no standard chemotherapeutic regimen for metastatic

\footnotetext{
*Correspondence: Dr Y-H Im; E-mail: imyh00@skku.edu

${ }^{5}$ These authors contributed equally in this study.

Received 4 May 20I0; revised 28 June 20I0; accepted 6 July 20I0; published online 10 August 2010
}

oesophageal SCCA; hence, various kinds of chemotherapeutic regimens have been investigated in an attempt to prolong survival and improve the quality of life. 5-Fluorouracil (5-FU), cisplatin, and epirubicin are widely used chemotherapeutic agents for gastric and oesophageal cancers worldwide (Kim et al, 1993; Ross et al, 2002). One of the most commonly used regimens as first-line chemotherapy in metastatic oesophageal cancer is the combination of cisplatin and a 5-FU continuous infusion, with response rates (RRs) ranging from 30 to $40 \%$ (Ilzuka et al, 1992; Bleiberg et al, 1997; Enzinger et al, 1999). However, the continuous infusion of 5-FU and cisplatin combination therapy requires indwelling venous access, which may lead to venous thrombosis and sepsis, hence making therapy burdensome to the patient with significant toxicity.

Capecitabine (Xeloda, Hoffmann-La Roche Inc., Basel, CH, USA) is an oral fluoropyrimidine prodrug that is transformed to $\mathrm{FU}$ in several steps, the last of which is conversion of $5^{\prime}$-deoxy-5fluorouridine to FU by thymidine phosphorylase (TP). In patients with advanced oesophagogastric cancer, capecitabine combinations 
have generally shown good antitumour activity and highlight the potential of capecitabine as a replacement for infusional 5-FU (Cunningham et al, 2008; Kang et al, 2009). These results have shown that capecitabine is not inferior to FU. In addition, fluropyrimidine-related adverse events were similar in the capecitabine and FU groups. Thus, considering troublesome venous access, capecitabine may be a good substitute for 5-FU.

A phase II trial of our previous study tested the safety and efficacy of capecitabine $\left(1250 \mathrm{mg} \mathrm{m}^{-2}\right.$ twice daily for 2 weeks) with cisplatin $\left(60 \mathrm{mg} \mathrm{m}^{-2}\right.$ on day 1 in 3 -week cycles; capecitabine and cisplatin (XP)) for chemonaive oesophageal SCCA and reported promising results with RRs of $57.8 \%$ and a tolerable toxicity profile (Lee et al, 2008), suggesting that a combination of capecitabine and platinum is one of the most effective regimens for the treatment of advanced oesophageal cancer. Capecitabine was designed to take advantage of the increased levels of TP observed in tumours as opposed to normal tissue, potentially allowing for selective toxicity in tumours (Van Cutsem et al, 2001). Elevated levels of TP are associated with tumour aggressiveness and poor prognosis (Bronckaers et al, 2009). 5-FU is then either degraded by dihydropyrimidine dehydrogenase (DPD) or anabolised to fluorodeoxyuridylate, which inhibits thymidylate synthase (TS). Thymidylate synthase, the target enzyme of 5-FU, has been shown to be an independent prognostic marker of 5-FU chemotherapy in gastrointestinal tumours (Lenz et al, 1996, 1998; Shirota et al, 2001). Meropol et al (2006) evaluated TP, TS, and DPD for their ability to predict a response to capecitabine when used in a firstline metastatic setting, in an attempt to identify patients who may have an altered response to a capecitabine/irinotecan regimen. The role of TP and TS to identify predictive markers for the treatment response to XP in oesophageal SCCA has not been defined.

Platinum compounds are additional key drugs in the treatment of oesophageal SCCA. Resistance to platinum agents has been attributed to enhanced tolerance to platinum DNA adducts, decreased drug accumulation, and enhanced DNA repair. Impairment of the DNA repair mechanism is important in the resistance to cisplatin. The destruction of cells by cisplatin requires binding of the drug to DNA and the creation of platinum-DNA adducts. Some of these adducts establish covalent cross-linking between DNA strands, thereby inhibiting DNA replication. Nucleotide excision repair (NER) has a central role in DNA repair and proteins of the NER pathway are thought to repair DNA damage caused by platinum agents. The excision repair cross-complementation group 1 (ERCC1) enzyme has a rate-limiting role in the NER pathway that recognises and removes cisplatin-induced DNA adducts (Olaussen et al, 2006).

The significance of TS, TP, and ERCC1 expression in oesophageal SCCA has not been studied in patients who have been treated with capecitabine and platinum combination chemotherapy. In this study, we determined the protein expression of TS, TP, and ERCC1 by immunohistochemical (IHC) staining in pretreatment biopsies of oesophageal tumour tissue specimens from patients who received palliative first-line chemotherapy with XP combination chemotherapy. This study was conducted to evaluate the clinical implications of these proteins as biomarkers, which could predict the outcome of XP chemotherapy.

\section{PATIENTS AND METHODS}

\section{Patients}

A total of 113 patients with recurrent or metastatic oesophageal SCCA were treated with XP chemotherapy at the Samsung Medical Center between July 2003 and December 2007, of whom 72 had clinical data and paraffin tissue blocks available for immunohistochemistry of TS, TP, and ERCC1 in this analysis. The median duration of follow-up was 41 months, with a range of 18-70 months. Our study protocol was approved by the Institutional Review Board of Samsung Medical Center.

\section{Treatment}

Patients received cisplatin $\left(60 \mathrm{mg} \mathrm{m}^{-2}\right.$ intravenously on day 1) and capecitabine $\left(1250 \mathrm{mg} \mathrm{m}^{-2}\right.$ per dose orally twice a day on days 1-14). Treatment cycles were repeated every 3 weeks until documented disease progression, unacceptable toxicity, or patient refusal. The treatment response was evaluated every two cycles by RECIST, version 1.0.

\section{IHC staining and scoring}

Tumour specimens from patients who were diagnosed on the basis of histological samples were evaluated for biomarker analyses. Full specimen sections ( $4 \mu \mathrm{m}$ thick) were cut from paraffin blocks and mounted on adhesive-coated or charged glass slides. An IHC method was used to determine the level of protein expression for TS, TP, and ERCC1.

Immunohistochemical staining was performed using the streptavidin-biotin complex method and the TechMate 1000 automated staining system (DakoChemmate, Glostrup, Denmark). The primary antibodies and working dilutions used were as follows: TS mouse mAb (clone TS106/4H4B1, 1:50 dilution in Tris/EDTA buffer (pH 9.0); Zymed, San Francisco, CA, USA) and TP mouse mAb (PGF.44C, $1: 100$ dilution; NeoMarkers, Fremont, CA, USA). Deparaffinised sections were treated with $3 \%$ hydrogen peroxide in methanol for $10 \mathrm{~min}$ to inhibit endogenous peroxidase. Sections were immersed in $0.01 \mathrm{~m}$ citrate buffer $(\mathrm{pH} 6.0)$ and heated in a pressure cooker for $30 \mathrm{~min}$. Sections were then incubated with primary antibody for $50 \mathrm{~min}$ at room temperature. Each section was treated sequentially with a biotinylated secondary antibody (antimouse immunoglobulin) and a streptavidin-peroxidase complex (DakoChemmate). 3,3'-Diaminobenzidine tetrahydrochloride was used as a chromogen, and Mayer's haematoxylin counterstain was applied. Experiments on negative controls (isotype-matched irrelevant antibody) were run simultaneously.

For ERCC1 detection, mouse monoclonal antibody ERCC1 (8F1; NeoMarkers) was used at a dilution of $1: 200$ overnight in a humidified chamber. The primary antibody was visualised with an avidin-biotin complex (ABC) system (Dako, Carpinteria, CA, USA). Slides were washed in TBS, the relevant biotinylated goat antimouse IgG diluted at 1:100 was added, and the slides were incubated for $20 \mathrm{~min}$ at room temperature. The sections were washed again in TBS and incubated for $10 \mathrm{~min}$ in a solution of streptavidin-ABC-horseradish peroxidase diluted at $1: 100$. Colour was developed by adding 3,3'-diaminobenzidine tetrahydrochloride (Immunotech, Cedex, France). Finally, the sections were counterstained with Mayer's haematoxylin.

For each patient, three full-specimen sections were analysed, one slide each for TS, TP, and ERCC1. The slides were assessed without the knowledge of clinical outcome by two experienced pathologists. Thymidine synthase and TP protein expression levels were detected in both the cytoplasm and nucleus, and ERCC1 showed nuclear staining (Figure 1).

The intensity and extent of the IHC staining was graded on a quantitative scale from 0 to $100 \%$ in proportion, except for ERCC1, and on a semiquantitative scale from 0 to 3 , as follows: $0=$ no staining; $1=$ weak staining; $2=$ strong staining; and $3=$ very strong staining. The area of most intense staining was graded on a scale from 0 to 4 , where $0=$ no staining, $1=0-10 \%$ staining of tumour cells, $2=10-25 \%$ staining of tumour cells, $3=25-50 \%$ staining of tumour cells, and $4=>50 \%$ staining of tumour cells. A TS score was calculated by multiplying the intensity and extent of grades. Thymidine synthase scores $<6$ were considered low, and a score $>6$ was defined as high in this study. The same criteria were applied for TP and ERCC1 staining results. 
A

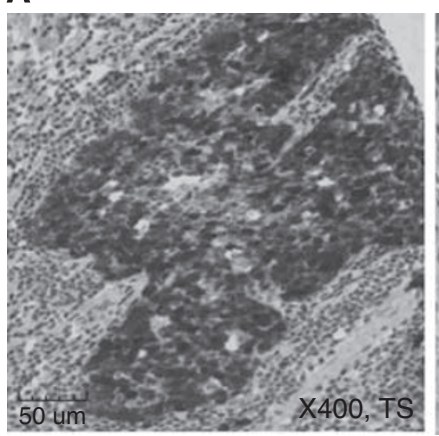

B

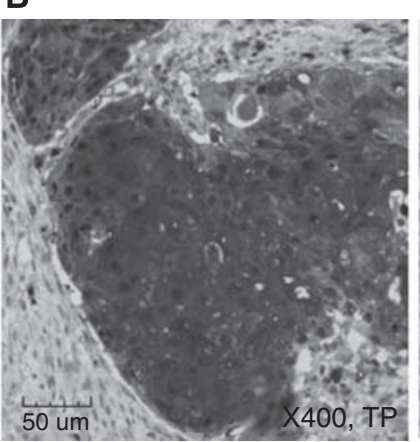

C

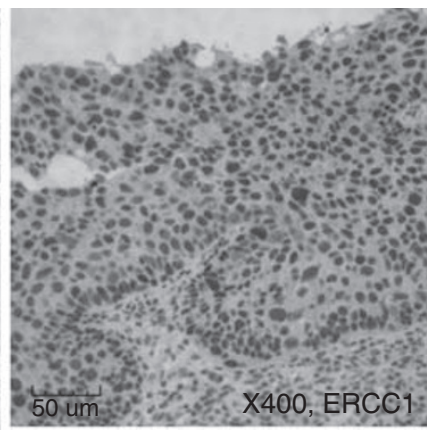

Figure I Immunohistochemical staining of TS, TP, and ERCCI in oesophageal squamous cell cancer. (A) High staining intensity for TS. The TS staining was predominantly a cytoplasmic granular pattern $(\times 400)$. (B) Positive sample with TP. The immunoreactivity was nuclear and cytoplasmic and it was highly expressed in mononuclear cells $(\times 400)$. (C) High expression of ERCCI. The ERCCI staining was strong and diffuse in the nucleus $(x 400)$.

\section{Statistical analysis}

The $\chi^{2}$ values of Fisher's exact tests, as indicated, were applied for the comparison of clinicopathological features of TS, TP, and ERCC1 tumours. Progression-free survival (PFS) was calculated from the first date of XP chemotherapy to the date of progression or death in the case of patients who died without progression. Overall survival (OS) was defined as the interval between the first date of chemotherapy and death or up to the date of the last followup evaluation. Both PFS and OS were calculated according to the Kaplan-Meier method. All reported $P$-values are two-sided. The contribution of prognostic variables to survival was analysed using the log-rank test for univariate analysis and the Cox proportional hazard model for multivariate analysis. The clinicopathological variables and expressions of TS, TP, and ERCC1 were examined for an association with the clinical outcome. The log-rank test was used to measure the association between TS, TP, and ERCC1, and survival. For quantitative analysis of TS and TP in terms of the extent of positive staining, the ANOVA test was used with response. We did not conduct survival analysis with quantitative results. Statistical significance was regarded as significant when $P<0.05$. All analyses were carried out using the Statistical Package for the Social Science (version17.0; SPSS Inc., Chicago, IL, USA) statistical software package.

\section{RESULTS}

\section{Patient characteristics and clinical outcome}

Between July 2003 and December 2007, 113 patients with advanced oesophageal cancer received palliative chemotherapy at our institution, with XP combination chemotherapy as a first-line treatment. Among these 113 patients, tissue samples of 72 patients were available for analysis of IHC staining of TS, TP, and ERCC1. The patient baseline characteristics are listed in Table 1. All patients had histologically proven SCCA of the oesophagus, and no patient had adenocarcinomas of the gastro-oesophageal junction. The median age was 62 years, with a range of $31-72$ years. In all, $25(34.7 \%)$ patients received adjuvant 5-FU and cisplatin (FP) chemotherapy after surgical resection; $68(94.4 \%)$ patients had an Eastern Cooperative Oncology Group performance status of $0-1$. The sites of metastasis included the lymph nodes $(76.4 \%)$, lung $(26.4 \%)$, liver $(18.1 \%)$, pleura $(11.1 \%)$, skin $(6.9 \%)$, and bone (5.6\%).

Of the 72 subjects who were considered assessable for response to therapy, 69 had an overall RR of $51.4 \%$, with 2 complete responses and 35 partial responses (51.4\%). Ten (14.5\%) patients had stable disease, but $22(30.6 \%)$ patients experienced clinical or radiographic evidence of disease progression. Three (4.2\%)
Table I Patients' characteristics $(n=72)$

\begin{tabular}{lcr}
\hline & $\begin{array}{c}\text { Number of } \\
\text { patients }(\mathbf{n}=\mathbf{7 2})\end{array}$ & $\%$ \\
\hline Sex & & \\
$\quad$ Male & 69 & 95.8 \\
$\quad$ Female & 3 & 4.2 \\
Age, median (range) & $(37-72)$ & \\
& & \\
Performance status & & 54.4 \\
O-I & 68 & 34.7 \\
2 & 4 & \\
Previous adjuvant FP chemotherapy & 25 & \\
XP cycle, median (range) & $4(1-12)$ & 76.4 \\
& & 26.4 \\
Metastatic sites & & 18.1 \\
Lymph nodes & 55 & 11.1 \\
Lung & 19 & 6.9 \\
Liver & 13 & 5.6 \\
Pleura & 8 & \\
Skin & 5 & \\
Bone & 4 & \\
\hline
\end{tabular}

Abbreviations: FP = fluouracil and cisplatin; $X P=$ capecitabine and cisplatin.

patients were not assessable because of loss to follow-up. The patient population had a median of four cycles of the XP regimen. The median time to PFS was 4.2 months (95\% confidence interval (CI) 3.0-5.4 months), and the median survival was 12.0 months (95\% CI 9.2-14.8 months). The median duration of follow-up was 41 months.

\section{IHC staining results of TS, TP, and ERCC1 expression}

Table 2 shows the IHC results of TS, TP, and ERCC1 expression according to intensity and proportion. The number and percentage of patients who had low scores are shown.

\section{Relationship between treatment response and biological marker expression}

The association between TS, TP, and ERCC1, and response to XP, as measured by IHC, is listed in Table 3. A high TS proportion, high TS intensity, and high TS score predicted a response (TS $<25 \%$ vs TS $\geqslant 25 \%$, RR 40.5 vs $59.5 \%, P=0.009$; TS intensity $0-1$ vs 2-3, RR 45.9 vs 54.1, $P=0.030$; TS score $\leqslant 6$ vs TS score $>6$, RR 40.5 vs $59.5 \%, P=0.005)$. High expression of TS and TP was also significantly correlated with treatment response to the XP regimen (high TS and TP vs low TS and TP, RR 54.1 vs $45.9 \%$, $P=0.022)$. High TS and TP expression with low ERCC1 expression 
$(0-2$ vs 3$)$ showed a significantly better response compared with other levels of expression (high TS and TP and low ERCC1 vs other levels of expression, RR 63.2 vs $36.8 \%, P=0.016$; Table 3, Figures $2-4)$. In addition, quantitative analysis, as well as TS and TP extents, showed significant correlation with responses (RR 3.77,

Table 2 TS, TP, and ERCCI by $I C$ staining

\begin{tabular}{|c|c|c|}
\hline & $\begin{array}{l}\text { Number of patients } \\
\text { with low scores }\end{array}$ & $\%$ \\
\hline \multicolumn{3}{|l|}{ TS } \\
\hline Area $(0-2$ vs $3-4)$ & $38 / 69$ & 55.1 \\
\hline Intensity $(0-1$ vs $2-3)$ & $40 / 69$ & 58.0 \\
\hline \multicolumn{3}{|l|}{$T P$} \\
\hline Area ( $0-2$ vs 3$)$ & $23 / 72$ & 31.9 \\
\hline Intensity ( $0-2$ vs 3 ) & $3 / 72$ & 4.2 \\
\hline \multicolumn{3}{|l|}{ ERCCl } \\
\hline Area $(0-2$ vs 3$)$ & $22 / 41$ & 53.7 \\
\hline Intensity (0-2 vs 3 ) & $|4 / 4|$ & 34.1 \\
\hline
\end{tabular}

$P<0.0001$ for TS extent (\%); RR 4.12, $P=0.045$ for TP extent by ANOVA test, Table 3).

\section{Univariate and multivariate analyses of factors associated with prognosis}

We investigated the prognostic significance of various clinicopathological factors in patients with metastatic oesophageal SCCA. The univariate analysis showed that a high TS score, high TP score, and low ERCC1 expression were important factors affecting the prolongation of PFS and OS. Multivariate analysis was performed on capecitabine biological markers separately and with the platinum markers combined. Strong ERCC1 expression and a low TS score were identified as independent risk factors for PFS (hazard ratio (HR) $10.71,95 \%$ CI $2.1-54.7, P=0.004$ for strong ERCC1 expression; and HR 2.9; 95\% CI 1.0-7.9, $P=0.044$ for a low TS score). Strong ERCC1 expression was the only unfavourable independent determinant for OS (HR 3.73; 95\% CI 1.39-10.0; Table 4).

\section{DISCUSSION}

Thymidine synthase and TP have a key role in 5-FU resistance. Thymidine synthase, an essential enzyme needed for DNA synthesis, is the target enzyme of 5-FU. A number of studies have

Table 3 Association between TS, TP, and ERCC-I and response (semiquantitative and quantitative analysis)

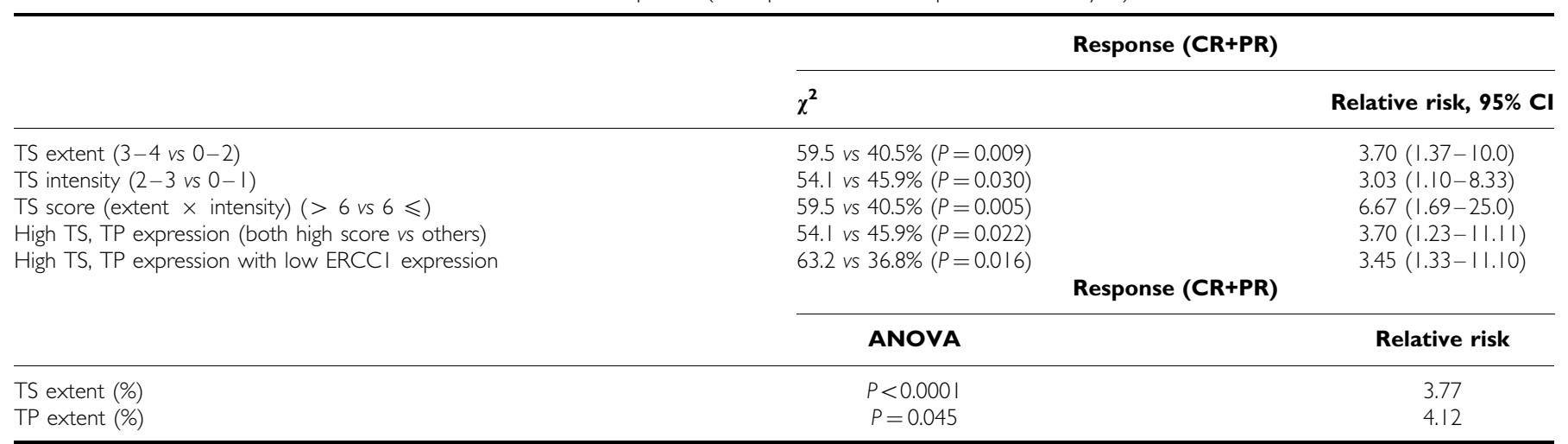

Abbreviations: $A N O V A=$ analysis of variance; $C l=$ confidence interval; $C R=$ complete response; $E R C C I=$ excision repair cross-complementation group I; $P R=$ partial response; $\mathrm{TP}=$ thymidine phosphorylase; $\mathrm{TS}=$ thymidine synthase. Intensity was graded on a semiquantitative scale from 0 to 3 , where $0=$ no staining, $I=$ weak staining, $2=$ strong staining, and $3=$ very strong staining. The area of the most intense staining was graded on a scale from 0 to 4 , where $0=$ no staining, $I=$ staining of $0-10 \%$ of tumour cells, $2=10-25 \%$ of tumour cells, $3=$ staining of $25-50 \%$ of tumour cells, and $4=$ staining of $>50 \%$ of tumour cells. A TS score was calculated by multiplying the intensity and extent grades. TS scores $<6$ were considered low, and score $>6$ were defined as high in this study. The same criteria were applied for TP and ERCCI staining results.
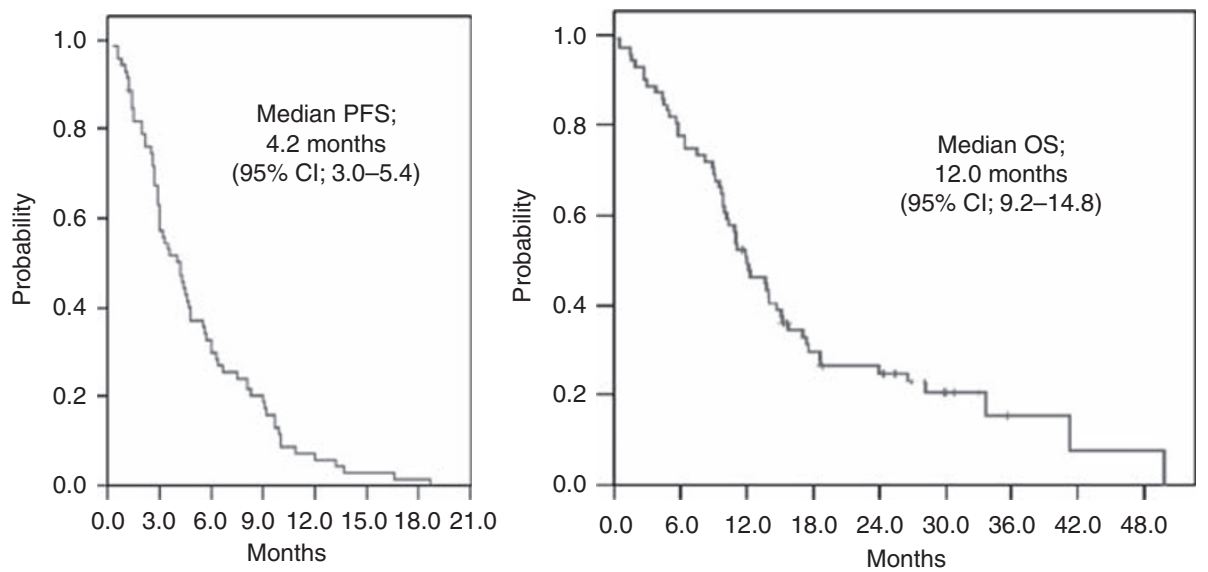

Figure 2 Kaplan-Meier curve for PFS and OS of XP in metastatic oesophageal SCCA. 

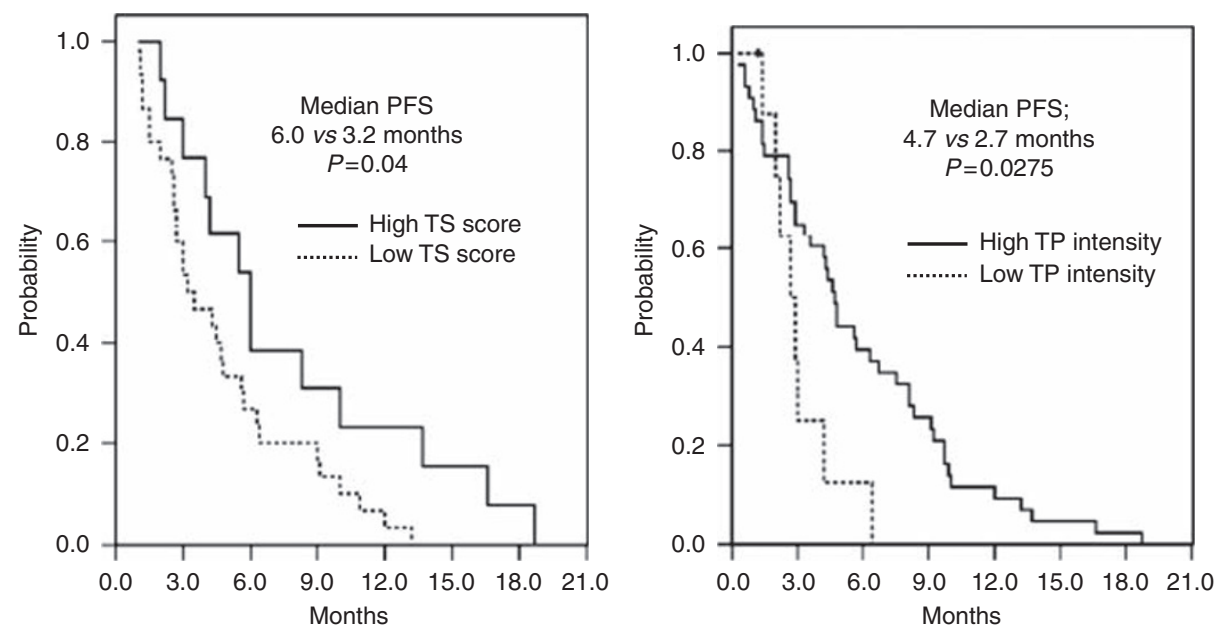

Figure 3 Thymidine synthase and TP expression and PFS.

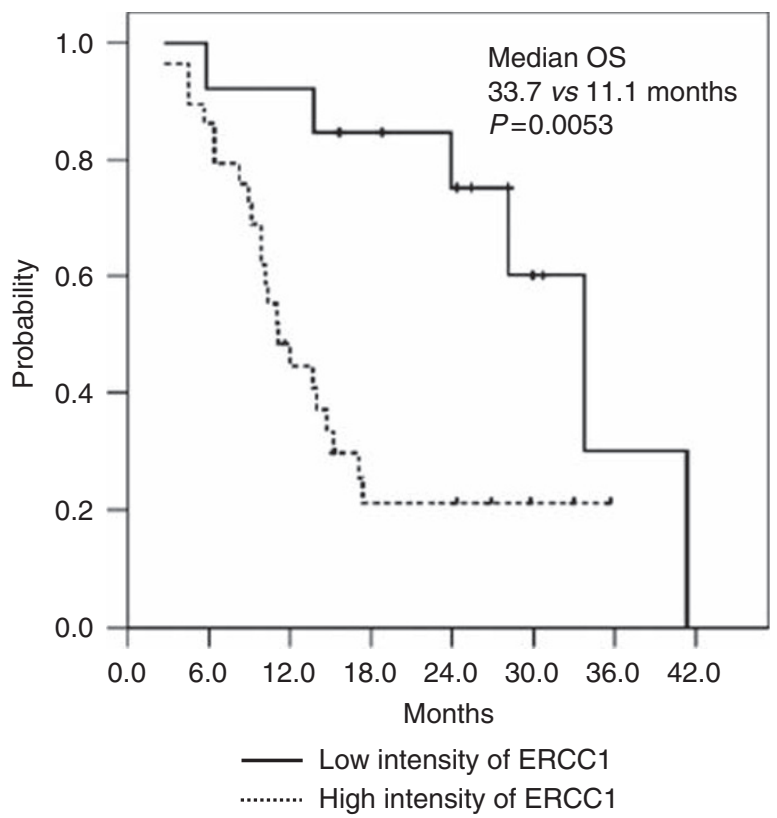

Figure 4 Excision repair cross-complementation group | expression and $O S$.

investigated TS expression and survival in colorectal cancer, but the data are conflicting (Popat et al, 2004). Some investigators have reported a survival benefit for patients with a high TS expression treated with 5-FU in adjuvant and metastatic colorectal cancer (Johnston et al, 1995; Edler et al, 2002; Soong and Diasio, 2005). In contrast to previous studies, different investigations have demonstrated a significant benefit in patients with low intratumoural TS levels (Allegra et al, 2002, 2003; Ciaparrone et al, 2006). Our results are consistent with previous reports and can be explained by the knowledge that high TS levels are most commonly associated with a responsiveness to 5-FU in patients with advanced disease. Although patients in this study were not randomly allocated to the study, exploratory analysis showed that the HR associated with capecitabine chemotherapy was 6.67 (95\% CI 1.69-25.0) for advanced oesophageal SCCA with a high TS score $(>6)$ compared with a low TS score.

Thymidine phosphorylase is the marker of a more aggressive and malignant tumour phenotype that has increased resistance to
Table 4 Multivariate Cox-regression hazard model for PFS and OS

\begin{tabular}{|c|c|c|c|c|c|c|c|c|}
\hline & \multicolumn{4}{|c|}{ PFS } & \multicolumn{4}{|c|}{ os } \\
\hline & $P$-value & HR & $95^{\circ}$ & $6 \mathrm{Cl}$ & $P$-value & HR & $95 \%$ & $\% \mathbf{C l}$ \\
\hline Low & 0.044 & 2.87 & 1.03 & 7.99 & 0.356 & 1.22 & 0.38 & 3.46 \\
\hline Strong ERCCI & 0.004 & $10.7 \mid$ & 2.10 & 54.71 & 0.009 & 3.73 & 1.39 & 10.04 \\
\hline Hepatic metastasis & 0.118 & 2.57 & 0.79 & 8.41 & 0.235 & 1.11 & 0.03 & 2.13 \\
\hline
\end{tabular}

Abbreviations: $\mathrm{Cl}=$ confidence interval; $\mathrm{ERCCI}=$ excision repair cross-complementation group I; $H R=$ hazard ratio; $O S=$ overall survival; $P F S=$ progression-free survival; TS = thymidine synthase.

cytotoxic agents because of the loss of apoptotic potential. This study also examined the impact of TP expression and its correlation with prognosis. Our study demonstrated a significant association between TP expression and clinical outcome. Thymidine phosphorylase expression was shown to be a positive predictive factor for FU response. However, the prognostic role of TP is not clear. Conflicting results have been reported in different studies. Li et al (2004) evaluated the association between TP-messenger RNA expression in tumour tissue and some prognostic factors (grade, tumour size, lymph node status, phase $S$ cellular fraction, ploidy, and clinical variables) with no statistically significant correlation. Yang et al (2002) analysed the expression of TP in 182 breast cancer patients with evidence of an association between TP expression, low grade, and low p53 expression. Thymidine phosphorylase-positive tumours seem to have a longer time to progression and OS, with a limited effect on angiogenesis. In contrast with these results, Tominaga et al (2002) published a retrospective study on TP expression in 650 samples of breast cancer. This study was not consistent with a previous report of an increase in tumour response and prolongation of OS in patients treated with $\mathrm{FU}$ and/or cisplatin-based chemotherapy for gastrointestinal cancer.

Excision repair cross-complementation group 1, the structurespecific DNA repair endonuclease responsible for $5^{\prime}$ incision, has a key role in the removal of adducts from genomic DNA through the NER pathway. We found that low expression of ERCC1 correlated significantly with a good response to systemic chemotherapy in patients with metastatic oesophageal SCCA. Kim et al (2008) reported that the low expression of ERCC1 correlated significantly with a good response to preoperative chemoradiotherapy in patients with oesophageal cancer, suggesting that ERCC1 expression may determine that the benefit of preoperative 
chemoradiotherapy may be more effective than immediate surgery in patients with a low ERCC1 expression. In contrast, immediate oesophagectomy may be as effective as preoperative chemoradiotherapy in patients with a high ERCC1 expression. These results were consistent with a prospective study in non-small-cell lung cancer, which showed that adjuvant platinum-based chemotherapy, compared with observation, significantly prolonged survival among patients with ERCC1-negative tumours (Olaussen et al, 2006). Jun et al (2008) compared their study of head and neck cancer treated with cisplatin-based concurrent chemoradiation with a study of non-small-cell lung cancer and showed that the proportion and pattern of ERCC1 expression varied according to tumour type. Multivariate analysis revealed that low expression of ERCC1 was an independent factor associated with a lower risk of cancer death. This result was also consistent with a previous report of an increase in tumour response and prolongation of OS in patients with oesophageal SCCA treated by cisplatin-based chemotherapy (Warnecke-Eberz et al, 2004; Joshi et al, 2005). Out data support the hypothesis that enhanced DNA repair decreases the benefit of platinum-based treatment.

If the combination of these three markers reflects the response and toxicity of the XP regimen in patients with metastatic oesophageal SCCA, we can predict who benefits from this regimen.
As only a relatively small proportion of patients seem to benefit from systemic chemotherapy in metastatic oesophageal SCCA, considerable effort has been directed towards finding biomarkers that can accurately predict tumour response. Currently, however, there is insufficient evidence to justify the incorporation of these or any other candidate predictive markers into routine clinical practice for the selection of oesophageal cancer patients to receive XP. If one could identify the patients likely to respond to a particular regimen, therapy could subsequently be tailored accordingly and the survival rate could be improved.

The limitation of this study included the inherent weakness of IHC staining, such as its semiquantitative nature, different antibodies used in various studies, inter or intraobserver variation, the variable cutoffs for TS, TP, and ERCC1 positivity, and the effects of tissue ageing. In addition, the number of patients was relatively small, and the study was retrospective in design. These correlative studies must be viewed as hypothesis generating and interpreted with caution because of the small sample sizes and limitation of a retrospective study.

In conclusion, this study has demonstrated that TS, TP, and ERCC1 expressions show promising markers for response and survival prediction of XP chemotherapy in patients with recurrent or metastatic oesophageal SCCA. Further prospective randomised studies are needed to confirm our results.

\section{REFERENCES}

Allegra CJ, Paik S, Colangelo LH, Parr AL, Kirsch I, Kim G, Klein P, Johnston PG, Wolmark N, Wieand HS (2003) Prognostic value of thymidylate synthase, Ki-67, and p53 in patients with Dukes' B and C colon cancer: a National Cancer Institute-National Surgical Adjuvant Breast and Bowel Project collaborative study. J Clin Oncol 21: 241-250

Allegra CJ, Parr AL, Wold LE, Mahoney MR, Sargent DJ, Johnston P, Klein P, Behan K, O'Connell MJ, Levitt R, Kugler JW, Tria Tirona M, Goldberg RM (2002) Investigation of the prognostic and predictive value of thymidylate synthase, $\mathrm{p} 53$, and $\mathrm{Ki}-67$ in patients with locally advanced colon cancer. J Clin Oncol 20: 1735-1743

Bleiberg H, Conroy T, Paillot B, Lacave AJ, Blijham G, Jacob JH, Bedenne L, Namer M, de Besi P, Gay F, Collette L, Sahmoud T (1997) Randomised phase II study of cisplatin and 5-fluorouracil (5-FU) versus cisplatin alone in advanced squamous cell oesophagela cancer. Eur J Cancer 33: $1216-1220$

Bronckaers A, Gago F, Balzarini J, Liekens S (2009) The dual role of thymidine phosphorylase in cancer development and chemotherapy. Med Res Rev 29: $903-953$

Ciaparrone M, Quirino M, Schinzari G, Zannoni G, Corsi DC, Vecchio FM, Cassano A, La Torre G, Barone C (2006) Predictive role of thymidylate synthase, dihydropyrimidine dehydrogenase and thymidine phosphorylase expression in colorectal cancer patients receiving adjuvant 5-fluorouracil. Oncology 70: 366-377

Cunningham D, Starling N, Rao S, Iveson T, Nicolson M, Coxon F, Middleton G, Daniel F, Oates J, Norman AR, Upper Gastrointestinal Clinical Studies Group of the National Cancer Research Institute of the United Kingdom (2008) Capecitabine and oxaliplatin for advanced esophagogastric cancer. $N$ Engl J Med 358: 36-46

Edler D, Glimelius B, Hallström M, Jakobsen A, Johnston PG, Magnusson I, Ragnhammar P, Blomgren H (2002) Thymidylate synthase expression in colorectal cancer: a prognostic and predictive marker of benefit from adjuvant fluorouracil-based chemotherapy. J Clin Oncol 20: 1721-1728

Enzinger PC, Ilson DH, Kelsen DP (1999) Chemotherapy in esophageal cancer. Semin Oncol 26: $12-20$

Ilzuka T, Kakegawa T, Ide H, Ando N, Watanabe H, Tanka O, Takagi I, Isono K, Ishida K, Arimori M (1992) Phase II evaluation of cisplatin and 5-fluorouracil in advanced squamous cell carcinoma of the esophagus: a Japanese Esophageal Oncology Group Trial. Jpn J Clin Oncol 22: 172-176

Joshi MB, Shirota Y, Danenberg KD, Conlon DH, Salonga DS, Herndon 2nd JE, Danenberg PV, Harpole Jr DH (2005) High gene expression of TS1, GSTP1, and ERCC1 are risk factors for survival in patients treated with trimodality therapy for esophageal cancer. Clin Cancer Res 11: $2215-2221$

Johnston PG, Lenz HJ, Leichman CG, Danenberg KD, Allegra CJ, Danenberg PV, Leichman L (1995) Thymidylate synthase gene and protein expression correlate and are associated with response to 5-fluorouracil in human colorectal and gastric tumors. Cancer Res 55: 1407 - 1412

Jun HJ, Ahn MJ, Kim HS, Yi SY, Han J, Lee SK, Ahn YC, Jeong HS, Son YI, Baek JH, Park K (2008) ERCC1 expression as a predictive marker of squamous cell carcinoma of the head and neck treated with cisplatinbased concurrent chemoradiation. Br J Cancer 99: 167-172

Kamangar F, Dores GM, Anderson WF (2006) Patterns of cancer incidence, mortality, and prevalence across five continents: defining priorities to reduce cancer disparities in different geographic regions of the world. J Clin Oncol 24: 2137-2150

Kang YK, Kang WK, Shin DB, Chen J, Xiong J, Wang J, Lichinitser M, Guan Z, Khasanov R, Zheng L, Philco-Salas M, Suarez T, Santamaria J, Forster G, McCloud PI (2009) Capecitabine/cisplatin versus 5-fluorouracil/cisplatin as first-line therapy in patients with advanced gastric cancer: a randomised phase III noninferiority trial. Ann Oncol 20: $666-673$

Kim MK, Cho KJ, Kwon GY, Park SI, Kim YH, Kim JH, Song HY, Shin JH, Jung HY, Lee GH, Choi KD, Kim SB (2008) Patients with ERCC1-negative locally advanced esophageal cancers may benefit from preoperative chemoradiotherapy. Clin Cancer Res 14: 4225-4231

Kim NK, Park YS, Heo DS, Suh C, Kim SY, Park KC, Kang YK, Shin DB, Kim HT, Kim HJ (1993) A phase III randomized study of 5-fluorouracil and cisplatin versus 5 -fluorouracil, doxorubicin, and mitomycin $\mathrm{C}$ versus 5 -fluorouracil alone in the treatment of advanced gastric cancer. Cancer 71: $3813-3818$

Lee J, Im YH, Cho EY, Hong YS, Lee HR, Kim HS, Kim MJ, Kim K, Kang WK, Park K, Shim YM (2008) A phase II study of capecitabine and cisplatin (XP) as first-line chemotherapy in patients with advanced esophageal squamous cell carcinoma. Cancer Chemother Pharmacol 62: $77-84$

Lenz HJ, Hayashi K, Salonga D, Danenberg KD, Danenberg PV, Metzger R, Banerjee D, Bertino JR, Groshen S, Leichman LP, Leichman CG (1998) p53 point mutations and thymidylate synthase messenger RNA levels in disseminated colorectal cancer: an analysis of response and survival. Clin Cancer Res 4: 1243-1250

Lenz HJ, Leichman CG, Danenberg KD, Danenberg PV, Groshen S, Cohen H, Laine L, Crookes P, Silberman H, Baranda J, Garcia Y, Li J, Leichman L (1996) Thymidylate synthase mRNA level in adenocarcinoma of the stomach: a predictor for primary tumor response and overall survival. J Clin Oncol 14: $176-182$ 
Li H, Suo Z, Zhang Y, Risberg B, Karlsson MG, Villman K, Nesland JM (2004) The prognostic significance of thymidine phosphorylase, thymidylate synthase and dihydropyrimidine dehydrogenase mRNA expressions in breast carcinomas. Histol Histopathol 19: 129-136

Meropol NJ, Gold PJ, Diasio RB, Andria M, Dhami M, Godfrey T, Kovatich AJ, Lund KA, Mitchell E, Schwarting R (2006) Thymidine phosphorylase expression is associated with response to capecitabine plus irinotecan in patients with metastatic colorectal cancer. J Clin Oncol 24: 4069-4077

Olaussen KA, Dunant A, Fouret P, Brambilla E, André F, Haddad V, Taranchon E, Filipits M, Pirker R, Popper HH, Stahel R, Sabatier L, Pignon JP, Tursz T, Le Chevalier T, Soria JC, IALT Bio Investigators (2006) DNA repair by ERCC1 in non-small-cell lung cancer and cisplatin-based adjuvant chemotherapy. $N$ Engl J Med 355: 983-991

Popat S, Matakidou A, Houlston RS (2004) Thymidylate synthase expression and prognosis in colorectal cancer: a systematic review and meta-analysis. J Clin Oncol 22: 529-536

Ross P, Nicolson M, Cunningham D, Valle J, Seymour M, Harper P, Price T, Anderson H, Iveson T, Hickish T, Lofts F, Norman A (2002) Prospective randomized trial comparing mitomycin, cisplatin, and protracted venousinfusion fluorouracil (PVI 5-FU) with epirubicin, cisplatin, and PVI 5-FU in advanced esophagogastric cancer. J Clin Oncol 20: 1996-2004

Shirota Y, Stoehlmacher J, Brabender J, Xiong YP, Uetake H, Danengerg KD, Groshen S, Tsao-Wei DD, Danenberg PV, Lenz HJ (2001) ERCC1 and thymidylate synthase mRNA levels predict survival for colorectal cancer patients receiving combination oxaliplatin and fluorouracil chemotherapy. J Clin Oncol 19: 4298-4304
Soong R, Diasio RB (2005) Advances and challenges in fluoropyrimidine pharmacogenomics and pharmacogenetics. Pharmacogenomics 6: $835-847$

Tebbutt NC, Cummins MM, Sourjina T, Strickland A, Van Hazel G, Ganju V, Gibbs D, Stockler M, Gebski V, Zalcberg J, on behalf of the Australasian Gastro-Intestinal Trials Group (2010) Randomised, non-comparative phase II study of weekly docetaxel with cisplatin and 5-fluorouracil or with capecitabine in oesophagogastic cancer: the AGITG ATTAX trial. Br J Cancer 102: 475-481

Tominaga T, Toi M, Ohashi Y, Abe O (2002) Prognostic and predictive value of thymidine phosphorylase activity in early-stage breast cancer patients. Clin Breast Cancer 3: 55-64

Van Cutsem E, Cunningham D, Hoff PM, Maroun J (2001) Thymidine phosphorylase (TP) activation: convenience through innovation. Oncologist 6(Suppl 4): 1-2

Warnecke-Eberz U, Metzger R, Miyazono F, Baldus SE, Neiss S, Brabender J, Schaefer H, Doerfler W, Bollschweiler E, Dienes HP, Mueller RP, danengerg PV, Doelscher AH, Schneider PM (2004) High specificity of quantitative excision repair cross-complementing 1 messenger RNA expression for prediction of minor histopathological response to neoadjuvant radiochemotherapy in esophageal cancer. Clin Cancer Res 10: $3794-3799$

Yang Q, Barbareschi M, Mori I, Mauri F, Muscarà M, Nakamura Y, Yoshimura G, Sakurai T, Caffo O, Galligioni E, Dalla Palma P, Kakudo K (2002) Prognostic value of thymidine phosphorylase expression in breast carcinoma. Int J Cancer 97: 512-517 